\title{
Towards a Generic Framework for Situated Collaborative Storytelling
}

\author{
Damian Hills Creativity and \\ Cognition Studio University of \\ Technology Sydney, Australia \\ +61-2-9514-4641 \\ damski@assimilate.net
}

\author{
Yusuf Pisan Creativity and \\ Cognition Studio University of \\ Technology Sydney, Australia \\ +61-2-9514-4478 \\ ypisan@it.uts.edu.au
}

\author{
Ernest Edmonds Creativity \\ and Cognition Studio University \\ of Technology Sydney, \\ Australia \\ +61-2-9514-4640
}

ernest@ernestedmonds.com

\begin{abstract}
How we assimilate stories into our common experiences and shape culture is the field of study known as narrative intelligence. By following these assumptions and investigating theories of conversation and rhetoric, this paper outlines a generic framework for a visual collaborative storytelling system that emphasises participatory narration and shared understanding in a situated context.
\end{abstract}

\section{Categories and Subject Descriptors}

H.5.1 Multimedia Information Systems

\section{General Terms}

Design, Experimentation, Human Factors, Theory.

\section{Keywords}

Interactive Digital Storytelling, Collaboration, Narrative Intelligence, Conversation Theory, Conversational Information System, Cognitive Information Retrieval

\section{INTRODUCTION}

Maintaining narrative coherence is one of the main difficulties faced in interactive storytelling system design. Interactive storytelling implies some form of participant control of the system and this creates a direct conflict with coherent delivery of narrative, therefore management of this relationship remains an important factor in any design. To deal with this problem, interactive storytelling research tends to focus on techniques of plot segment delivery including systems that are rule based [1], goal based [2] or those based on narrative functions constrained by a story context [3]. These systems attempt to automate narration based on participant choice with moderate success, yet they remain tightly coupled to their content and thus lack adaptability to larger or generic domains.

This paper proposes an alternative strategy that invites collaboration and shared narration allowing for a larger scope of generic themes in a visual storytelling context. By placing emphasis on participatory narration it assumes that narrative coherence is largely dependant on the collaborators themselves, however this in itself is no guarantee of a qualitative experience. By drawing upon certain theoretical assumptions a framework for a collaborative storytelling system is presented. This framework accounts for how narrative manifests in our community and the mental models we form from those influences. It can be broadly classed as a 'conversational information system' [4], or one that structures knowledge to manifest coherent collaborations by modelling the supportive processes involved during a group conversation. The system design supports the situated action [5] associated with oral and visual storytelling and the exchange of narrative themes. Through the enactment of conversation, participants can form a shared agreement surrounding several themes and reach a shared understanding.

To support the system design, several key theories are explored. These include our narrative intelligence, or our capacity to accrue canonical narrative themes through our communities and cultural experiences, followed by Conversation Theory (CT) [7], a generalised model of conversation and thematic sense-making, and then how mental models of narrative are exchanged and interpreted. Drawn from these theories a storytelling framework is developed as a 3-tiered unified architecture including, a database of narrative templates, a visual storytelling system based on CT and finally an expressive multi-participatory interface.

\section{NARRATIVE INTELLIGENCE}

The field of study known as narrative intelligence can directly inform the design of interactive narrative and storytelling systems. Asking how we assimilate narrative into our common experiences and form an understanding of each other and our experiential world is to understand something of our inherent narrative psychology. This area of research places narrative at the centre of human development and shapes our meaning and understanding of mind, where our intentional states are organized into a 'folk psychology'; an accrued set of canonical meanings that are extended or breached by story telling [6]. An inherent recognition of the canonical provides a design methodology for collaborative narrative systems by aligning familiar narrative themes or templates with the mental models that participants develop through cultural practices. However, cultural familiarity is often transformed by the unexpected event or outcome. This is a communal process where a sense of belonging to a shared social environment, aids the balance between the canonical and unexpected deviations. An understanding of community and its intersubjective processes can point to methods of representing knowledge that manifests thematic sense-making and narrative 
coherence. A system that represents knowledge in this way, would mediate the processes through the typical way communities exchange narrative knowledge, and that is through embodied conversation.

\section{CONVERSATION THEORY}

In mid-70s, cybernetician and psychologist Gordon Pask set out to develop a general theory of human communication and social interaction he named, Conversation Theory (CT). This theory is largely developed as a theory of learning that places conversation as central to its process and outlines a formal method of conversation.

Conversation is defined as a sense-making process or a negotiation of shared agreement given differing perspectives surrounding several themes. The framework developed for CT is essentially a self-organising system where sets of themes may be arranged coherently or whole leading to eventual action or procedures that clarify the context. This defines conversation based on two distinct levels, description of knowing 'why' (cognitive or conceptual) and knowing 'how' (procedural or performative) [7]. CT is a generalised model for representing the structure of conceptual and procedural knowledge and it is this distinction that allows it to create an adaptable framework that is open to collective viewpoint and interpretation.

Murray [9] defines procedurality as one of four fundamental properties of the digital computing medium. The others are spatial, participatory and encylcopedic. CT is able to embody all these properties, with the latter three as representations of our conceptual knowledge, and procedurality exhibiting the rules in parallel. In this sense, CT offers a model to combine conceptual and procedural knowledge in a collectively constructed system that is flexible and domain independent. For this reason, it may serve as a model for collaborative narrative and storytelling.

The central feature of CT is the construction of the entailment mesh. These are concept networks that embed interrelated knowledge, such that each concept that can be explained or understood by its relationship to at least two others. Such networks are said to introduce cyclicity and achieve a conceptual coherence. Cycling in this manner provides a rich set of possible narrative structures within the entailment structure. Scott [10] shows how expository narrative can be applied to CT yet does not rule out that various forms of narrative genre may also be applied:

'What is needed, and what is provided in Pask's sub-theory of conversational domains, is a canonical way of modelling the structure of knowledge content such that all possible narrative structures may be revealed and articulated as particular forms.'

Pask himself was a keen dramatist and eluded to the possibility of collaborative storytelling by discussing Koelster's [11] theory of creativity, in which he draws strong parallels with CT, explaining that his ideas involve characterisation, or how an audience of a play might relate themselves to the characters and "thus enact and extrapolate the plot in their own mind.". This refers to the fact that the interpretive affordances of the system are of significant influence in the meaning generated by participants and should be a major design consideration for narrative systems. Mateas also discusses this idea in his notion of Expressive AI, where the procedural processes although intentionally crafted by the author are open to interpretation [12]. This is a rhetorical strategy that appeals to the cultural familiarity of the participants and essentially aims to balance viewpoints and invite reflection. Bogost [13] identifies this as procedural rhetoric, an approach that would suit collaborative works.

\section{MENTAL MODELS}

By placing emphasis on human intention in narrative, a distinction can be made between narration (sjuzet) and story plot (fabula) [14]. The assumption is the enactment of narrative, or the act of the telling through conversation, is the means by which mental models are formed and shared. These models, as Vico suggests, are layers of constructed metaphors that are shaped from our existing conceptual structures [15]. Essentially, any knowledge structure is a result of metaphorical associations from existing conceptual domains that are said to originate from our affective experiences. This explicates the origins of narrative as an inductive process drawn directly from events and outcomes from our experiential life.

Storytelling systems can benefit from these assumptions by offering a rich set of events and relationships that appeal to existing metaphors and accrued knowledge patterns. Semantic relations can connect the various contextual meanings and form a larger pattern of relational knowledge for mental models to form. Semantic inference models hold the scalability and flexibility needed for systems to encompass larger domains and may ultimately suit generic collaborative environments. Typically, the basis of the semantic model is a network of interrelated plot segments or events. The semantic network acts as a story ontology that forms meaning from its relations rather than just from the events alone. Interactive storytelling researchers [16],[17] exploit this fact by using ontology to act as a template that may be permutated into new stories by participants in the narrative. Such templates are desirable by offering an ontology of contextual relationships that can assist in the assembly of a story world. The context in the story ontology allows for a multi-representation [18] of thematic ideas that are drawn from a general story corpus. Systems that use common sense reasoning can create the basis of the ontological world in which the narrative can take place, thus allowing the collaborative themes to emerge.

However, other researchers [19] point to the flexibility of rhetorical relations to connect narrative events. This allows not only the semantic relation of the text, but also the broader sense of ideas and concepts related to the text. Rhetorical strategies like these appeal directly to our mental models of narrative by reflecting points of view during active engagement with the systems processes. Returning to Bogost's notion of procedural rhetoric, the processes reflect a point of view that sits in-between the participant's subjectivity and the rules that make up the system [13]. In a collaborative context rhetorical relations could foster agreement by aligning participants views of the story event relationships and allowing them to cycle through the processes to understand the point of view of the current narrator. In Pask's CT we find the ability to cycle through procedural tasks until there is a shared agreement, yet it also allows and accommodates differing views, and this might be how the narrator/participant breaches the canonical form and presents a new narrative from an existing template.

\section{STORYTELLING FRAMEWORK}

The general aim of the framework is to allow for a process of distributed narration and authorship. Combining aspects of our inherent narrative intelligence and the mental models we form 
around stories, this framework is be flexible enough to incorporate a large domain of existing narrative templates containing sets of semantic and rhetorical relationships. This combines CT entailment structures and the Rhetorical Structure Theory (RST) ontology model as developed by Nakasone and Ishizuka [19], where each template is mapped into Pask's cognitive distinction of declarative and procedural knowledge. The entailment nets are activated as collections of themes that contribute to the narrative in question (why), while the rhetorical relations form the procedural or possible sequences of events that can take place (how). This is represented as a graph model that bootstraps CT entailment nets, that are inherently non-directional, with a directed graph of RST relations. Knowledge represented in entailment meshes is bootstrapped with semantic relations, these then refine the ambiguous asymmetric relations of the mesh [20]. The rhetorical relations offer procedural ways to navigate the context of the constructed entailment nets while the ambiguity is largely regulated by participants.

The proposed framework is constructed as a multi-tiered network architecture including, the database tier containing a preprocessed set of templates with recognizable narratives, the application tier consisting of two layers, storyworld and storyline that models the conceptual and procedural relationships, and finally a presentation tier that allows for collaborative ratings of the given procedures. (see Figure 1)

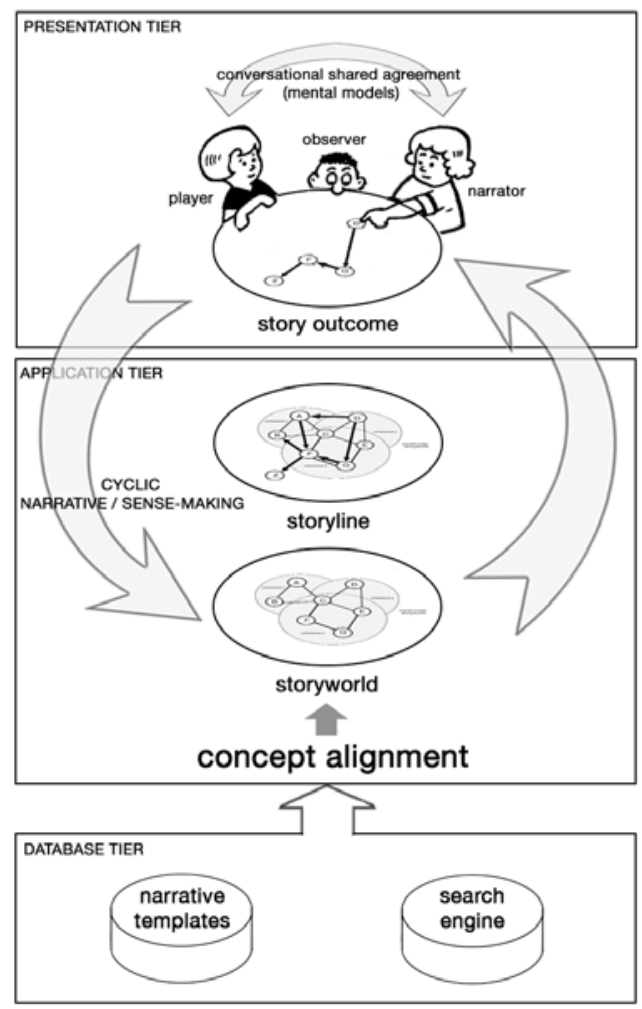

Figure 1. 3-tiered architecture

\subsection{Template Database Tier}

The database tier stores sets of story ontology templates containing predetermined associative concept relations, used to service the application tier. The use of ontology templates is a common technique [16], [17] and these are typically stored as semantic web languages, OWL or RDF, for their flexibility and computability. However a database supporting multi-dimensional data representations [22], may be suitable to support applications that implement conversational knowledge representations [21]. Pre-processed template concepts are semantically aligned with the themes selected by participants. The concepts can then be easily associated with others in the network and their context inferred. The context in the templates is derived from common-sense databases [23] where contextual knowledge are representations of facts from every-day life, including relationships that are spatial, temporal and causal. Common sense knowledge has a wide and shallow domain as opposed to expert knowledge that is deep. For this reason, it is applicable to a general storytelling corpus where typical or multi-representational themes can be assembled.

\subsection{Application Tier}

The application tier consists of two layers, firstly the Storyworld constructed as CT entailment structures, and the Storyline forming the possible procedures of that world structured as RST relationships.

\subsubsection{Storyworld Construction}

The storyworld concepts are the narrative themes that are assembled by participants selected from narrative templates and arranged into CT entailment structures. Entailment networks are collections of themes that make up the contextual relationships and contain common or logically inferred relationships. In this model it is seen as the context for the story world.

Techniques of clustering and coherence checking are fundamental to participatory conversational networks. Ford [24] states the desire for a flexible collaborative system that allows for negotiated shared agreement. The main advantage of the entailment structure is its ability to form coherence. Given a network of three or more themes $(A, B, C)$, the context of theme A entails the context of $B$ and $C$. This simple idea shows that each theme can be explained on its relationship to at least two others, such a network is known as coherent. (see Figure 2)

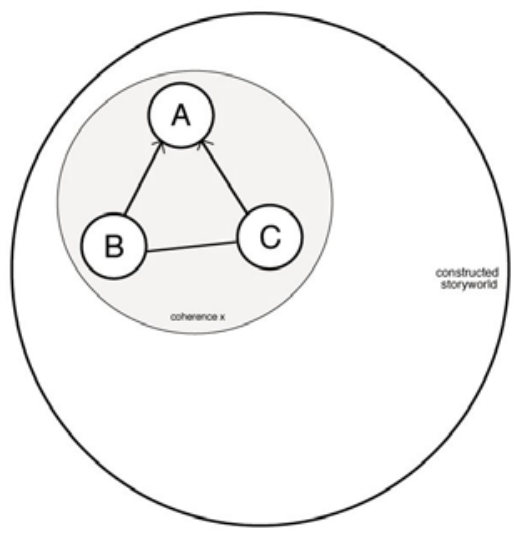

Figure 2. Simple Entailment Structure

A coherent entailment network is considered 'operationally closed', or cyclic, in its dependency. This is advantageous for collaboration as it allows participants to adopt several viewpoints or perspectives. It also collectively shapes the network's meaning. Participants construct the narrative themes in this manner to form any arbitrary number of coherent networks that are assigned 
ratings dependent on the contextual strength of their associated theme relation. (see Figure 3)

In CT, entailment networks allow for the process of pruning, or reducing, the ambiguity by merging related themes or separating them by analogy. This model allows for this process to be conducted in real time through collective participation reducing the complexity of relations, and assisting with visualisation.

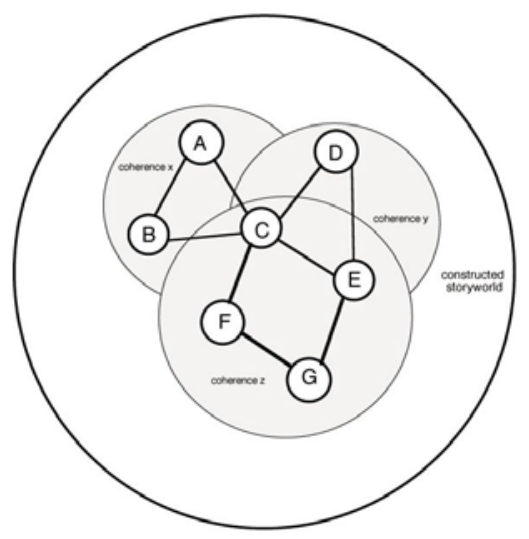

Figure 3. Storyworld Construction

The construction of the storyworld is a continual sense-making process where collected thematic ideas may be assembled collaboratively. CT entailment networks, story ontology templates and common sense knowledge all contribute to this process. However for the requirement of storytelling, this context alone cannot explain how the narrative manifests. This leads on to the selection of procedural ways the themes may be arranged into one or more storylines.

\subsubsection{Storyline Construction}

The storyline is the possible event sequences that may take place. These are the relationships between the assembled themes in the form of RST relations and make up the procedural content of the narrative. Pask defined these procedures as task structures or the operational methods by which to understand the context of the network. The CT entailment structures allow for any number of tasks and numerous ways to perform them. In this storytelling model, the procedural task is translated as one possibility of a storyline. (see Figure 4)

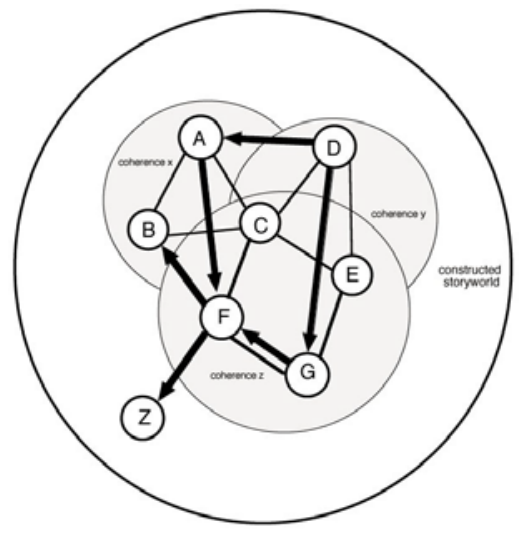

Figure 4. Storyline Construction

\subsection{Presentation/Interface Tier}

The presentation tier consists of a multi-participatory interface that facilitates the collective choice of procedural content as assembled by the application tier. The key requirements for this interface would be firstly to allow participants to seed thematic content to be aligned with the template database and placed within the storyworld, and secondly to facilitate the selection of procedural events based on those assembled from the storyline. The interface should also render visible the most salient narrative structures from the temple database yet cater for a broader range of narratives that may suit the thematic context. These narratives are considered the fittest based on collective ratings calculated from several metrics including, its conceptual semantic relevance, its narrative event relationships and most importantly, the relevance given by the participants.

The interface aids collaboration by reflecting the intentions of participants with the systems processes. Rendering the salient narratives also expresses the processes associated with them. This is a form of expressive processing [25] that looks at the relationship between the systems processes and the surface level interaction with the participants. This approach is central to the systems relationship with its collaborators as it supports the role the participant wishes to play or enact through conversation by verbal, non-verbal or gestural means. Revealing those processes at the surface level allows participants to switch roles dynamically between narrator, player and observer. Those situated actions are also supported by the interface and system states to promote the shared outcomes and consensus.

\subsection{Application}

The following outlines an example of how the framework is applied to the collaborative storytelling process. The tiered architecture is supported by a multi-user touch table that promotes gesture, verbal and non-verbal communication. The interface manages a collaborative environment that allows participants to visually construct narratives in an abstract virtual space. Narrative themes are assigned visual behaviours that are regulated by collective ratings. The system is a visual search engine that aligns participant themes with template stories drawn from a database of mythology and folklore [26] that springboards new collaborative narratives based on those themes. (see Figure 5.)

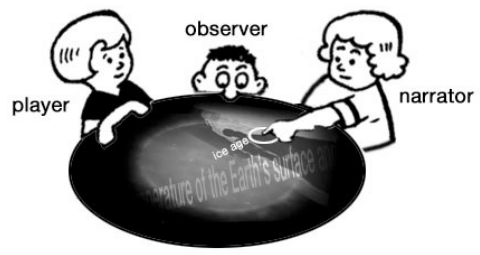

Figure 5. Visual story construction

The corpus has been transformed into a story grammar to ascertain common story relations such as narrative outcomes or events. Each theme is associated with a series of analogies with weightings that allow search themes to be aligned contextually. This example shows a narrative from the database:

A719.2. After world catastrophe, new sun reappears and starts new epoch. S. Am. Indian (Chiriguano): MÇtraux RMLP XXXIII $154 \mathrm{ff}$. 
In this case, a centuries old Indian myth surrounding a 'catastrophe' can be associated with modern issues such as 'global warming' and presented as a symbolic event in the template's narrative structure. Common sense databases contain many such analogous relationships that can associate everyday meanings with the example themes. Following CT's entailment structures, the themes contain derivable relationships are supported by at least two others, in this case 'catastrophe' may be supported by two other themes, 'nature' and 'war' that stem from other myths in the corpus. These themes are then added to the storyworld to further episodic content to the existing narrative, forming subplots or differing outcomes that break with the canonical. The assembled themes then reveal the possible procedural outcomes of the storyline, here the RST relationships can describe how events fit into the collaborative scheme, as 'war' might be seen as an episodic elaboration to the 'catastrophe' theme. Since CT entailment structures are cyclic, narrative themes may be revisited for further elaborations or forming new storylines. (Figure 6)

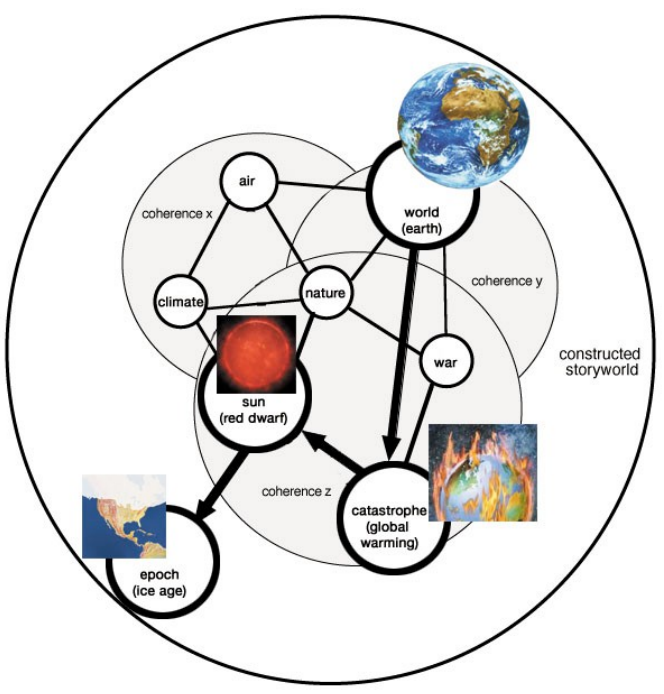

Figure 6. Example - 'After world catastrophe, new sun reappears and starts new epoch.'

The collaborative process takes place in a situated context where gesture and conversation are mediated by the themes presented and allows participants to shift between roles as narrator, player and observer while maintaining a visual coherence. The example shows how a modern theme is derived from the myth or a hidden truth from our cultural history that is reinterpreted and enacted. It is this action that essentially promotes the shared understanding and intersubjective processes that complete the story.

\section{CONCLUSION}

This framework takes a broader view of its potential application for collaboration, one that places a focus on face-to-face group situations and emphasises how our mental models and intentions are understood by others, and not necessarily by the system itself. This supports situated action, where the enactment of narrative by participants is mediated by the conversational knowledge generated by the system. Such systems look to holistic paradigms of information retrieval and exchange that combine logical and affective thought to deliver a coherent experience. The systems design can favour such schemes by offering a unified architecture, starting with narrative templates that are recognisable from our cultural backgrounds, feeding up into a real time process of shared negotiation of themes through embodied conversation that is mediated by a novel interface. The interface should take on an expressive appeal that renders visible its inner processes and allows collaborators to shift roles from narrator, player and/or observer.

Modelling the thematic relationships in a conversational form, such as Pask's entailment structures, allows for a cyclic interpretation and may be suitable to a range of narrative styles in a domain independent and scalable way. CT entailment structures can connect concepts using common sense and rhetorical relationships that extend the meaning beyond the literal boundaries creating a link with our cultural narratives that the system itself cannot capture. This holistic aim of negotiating agreement and sense-making was largely the motivation of Pask's CT and there is much relevance of his work to networked collaboration, as there is to interactive narrative research.

\section{ACKNOWLEDGMENTS}

The authors of this paper acknowledge the support of the Australasian CRC for Interaction Design (ACID) through the Cooperative Research Centre Program of the Australian Government's Department of Innovation, Industry, Science and Research.

\section{REFERENCES}

[1] Crawford, C. Chris Crawford on Interactive Storytelling (New Riders Games). New Riders Games, 2004.

[2] Cavazza, M., Charles, F. and Mead, S.J. Interacting with virtual characters in interactive storytelling. Proceedings of the first international joint conference on Autonomous agents and multiagent systems: part 1. 2002, 318-325.

[3] Mateas, M. and Stern, A. Façade: An Experiment in Building a Fully-Realized Interactive Drama. Game Developers Conference, Game Design track. 2003.

[4] Ford, N. "Conversational" information systems - Extending educational informatics support for the web-based learner. 2004.

[5] Suchman, L.A. Plans and Situated Actions: The Problem of Human-Machine Communication. Cambridge University Press, 1987.

[6] Bruner, J. Acts of Meaning, 1991.

[7] Pask, G. Conversation theory: Applications in education and epistemology. Elsevier, Amsterdam, 1976.

[8] Pask, G. Conversation, cognition and learning : a cybernetic theory and methodology. Elsevier, Amsterdam ; New York, 1975.

[9] Murray, J.H. Hamlet on the Holodeck: The Future of Narrative in Cyberspace. The Free Press New York, NY, USA, 1997.

[10] Scott, B. Knowledge content and narrative structure. in Lyn Pemberton, S.S. ed. Words on the web : computer mediated communication / edited by Lyn Pemberton, Simon Shurville., 2000.

[11] Koestler, A. 1964, The Act of Creation, Hutchinson.

[12] Mateas, M. Expressive AI: A Hybrid Art and Science Practice. Leonardo, 34 (2). 2001, 147-153. 
[13] Bogost, I. Persuasive Games: The Expressive Power of Videogames. MIT Press, 2007.

[14] Bruner, J. Actual Minds, Possible Words. University Press, 1986.

[15] Danesi, M. Vico, metaphor, and the origin of language. Indiana University Press Bloomington, 1993.

[16] Tarau, P. and Figa, E. Knowledge-based conversational agents and virtual storytelling. Proceedings of the 2004 ACM symposium on Applied computing. 2004, 39-44.

[17] Tuffield, M.M., Millard, D.E. and Shadbolt, N.R. Ontological Approaches to Modelling Narrative. 2006.

[18] Mueller, E.T. Story understanding through multirepresentation model construction. Proceedings of the HLTNAACL 2003 workshop on Text meaning-Volume 9. 2003, 46-53.

[19] Nakasone, A. and Ishizuka, M. ISRST: An Interest Based Storytelling Model Using Rhetorical Relations. in Technologies for E-Learning and Digital Entertainment, 2007, 324-335.

[20] Heylighen, F. Bootstrapping knowledge representations From entailment meshes via semantic nets to learning webs
Francis Heylighen The Authors. Kybernetes, 30 (5/6). 2001, 691-725.

[21] Ford, N. New Cognitive Directions. New Directions in Cognitive Information Retrieval. 2005, 81-96.

[22] Chang, F., Dean, J., Ghemawat, S., Hsieh, W.C., Wallach, D.A., Burrows, M., Chandra, T., Fikes, A. and Gruber, R.E. Bigtable: A distributed storage system for structured data. Proceedings of the 7th USENIX Symposium on Operating Systems Design and Implementation (OSDI'06). 2006.

[23] Singh, P., Barry B., Liu,L. Teaching Machines About Everyday Life. BT Technology Journal, 22 (4). 2004, 227239.

[24] Ford, N. "Conversational” information systems. Journal of Documentation, 61 (3). 2005, 362-384.

[25] Wardrip-Fruin, N. Expressive Processing: On ProcessIntensive Literature and Digital Media, Brown University, 2006.

[26] Thompson, S. Motif-index of Folk-literature: A Classification of Narrative Elements in Folktales, Ballads, Myths, Fables, Mediaeval Romances, Exempla, Fabliaux, Jestbooks. Indiana University Press, 1997. 